\title{
Zeta-Functions Defined by Two Polynomials
}

\section{Kohji Matsumoto and Lin Weng}

\author{
Graduate School of Mathematics, Nagoya University, Chikusa-ku, Nagoya 464-8602, Japan
}

\section{Introduction}

Let $P(x), Q(x)$ be two non-zero polynomials with complex coefficients. Define the associated Dirichlet series $\zeta(s ; P, Q)$ by

$$
\zeta(s ; P, Q):=\sum_{n=1}^{\infty} \frac{P(n)}{Q(n)^{s}} .
$$

Clearly, $\zeta(s ; P, Q)$ is well-defined when Res is sufficiently large, provided that $Q(n) \neq 0$ for all $n=1,2,3, \ldots$, which in this paper we always assume.

It is the principal motivation of the present paper to study the analytic properties and the special values of $\zeta(s ; P, Q)$. However, we actually introduce the multi-variable series

$$
\zeta_{r}\left(\left(s_{1}, \ldots, s_{r}\right) ;\left(\alpha_{1}, \ldots, \alpha_{r}\right)\right):=\sum_{n=1}^{\infty}\left(n+\alpha_{1}\right)^{-s_{1}}\left(n+\alpha_{2}\right)^{-s_{2}} \ldots\left(n+\alpha_{r}\right)^{-s_{r}}
$$

where $s_{1}, \ldots, s_{r}$ are complex variables and $\alpha_{1}, \ldots, \alpha_{r} \in \mathbf{C} \backslash\{-1,-2, \ldots\}$, and study its analytic behavior. Note that $\left(n+\alpha_{j}\right)^{-s_{j}}=\exp \left(-s_{j} \log \left(n+\alpha_{j}\right)\right)$, where the branch of logarithm is fixed as $-\pi<\arg \left(n+\alpha_{j}\right) \leq \pi$. The properties of (1) can be easily deduced from those of (2), as we shall explain later.

Our interests in $\zeta(s ; P, Q)$ date back to the beginning of 90's. In order to evaluate precisely RaySinger analytic torsions for certain special symmetric spaces, during that time, the second author introduced $\zeta(s ; P, Q)$ and calculated the special values $\zeta(0 ; P, Q)$ and $\zeta^{\prime}(0 ; P, Q)$. However, the series $(1)$ is indeed a classical object: Various properties of the more general Dirichlet series

$$
\sum_{n_{1}=1}^{\infty} \ldots \sum_{n_{k}=1}^{\infty} \frac{P\left(n_{1}, \ldots, n_{k}\right)}{Q\left(n_{1}, \ldots, n_{k}\right)^{s}}
$$

where $P\left(x_{1}, \ldots, x_{k}\right), Q\left(x_{1}, \ldots, x_{k}\right)$ are two polynomials of $k$ indeterminants with complex coefficients, have been studied by many mathematicians. Under certain assumptions on the properties of $P$ and $Q$, Mellin $[\mathrm{Me} 1,2]$ and Mahler [Mah] established the meromorphic continuation of (3) to the whole complex plane $\mathbf{C}$, and studied the location of poles. This direction of research was revived in 80's. Many new results were obtained by, for instance, Cassou-Noguès [CN1,2,3], Sargos [S1,2], Lichtin [L1,2,3], Eie [E1,2] and Peter[P]. These authors were mainly concerned with the single variable series (3), though the included polynomials are of several indeterminants. However, Lichtin [L2] proposed the problem of investigating the multi-variable series

$$
\sum_{n_{1}=1}^{\infty} \ldots \sum_{n_{k}=1}^{\infty} \frac{P\left(n_{1}, \ldots, n_{k}\right)}{Q_{1}\left(n_{1}, \ldots, n_{k}\right)^{s_{1}} \ldots Q_{r}\left(n_{1}, \ldots, n_{k}\right)^{s_{r}}}
$$

where $P, Q_{1}, \ldots, Q_{r}$ are polynomials of $k$ indeterminants with complex coefficients. Lichtin indeed carried out such studies in his papers $[\mathrm{L} 4, \ldots, 7]$ when $Q_{1}, \ldots, Q_{r}$ are hypoelliptic. Under the assumption of the hypoellipticity, he proved in [L4] the meromorphic continuation of (4) to the whole $\mathbf{C}^{r}$. This result especially implies the meromorphic continuation of (2) and hence of (1). But Lichtin's method, based on the theory of $\mathcal{D}$-modules, is rather sophisticated and it is not clear how to deduce further explicit information from Lichtin's results.

In the first section of the present paper we prove the meromorphic continuation of (2) by a quite different method (Theorem A(i)(ii)), which obviously implies the meromorphic continuation of (1) (Theorem B). Moreover we prove that (1) is holomorphic at any non-positive integers (Theorem C). The starting 
point of our method is the Mellin-Barnes integral formula ((4) in Section 1). The prototype of the method can already be found in Mellin [M1] (see also Cassou-Noguès [C1,2]). Katsurada [K1,2] discovered that the Mellin-Barnes formula is useful to study the analytic behavior of double zeta sums. The first author [Mat1,2] generalized Katsurada's idea to obtain a proof of meromorphic continuation of Euler-Zagier multiple zeta sums. The method in this paper is a modification of the argument developed in [Mat1,2].

An advantage of our method is that it gives explicit information of the behavior of the multiple zeta functions $\zeta_{r}\left(\left(s_{1}, \ldots, s_{r}\right) ;\left(\alpha_{1}, \ldots, \alpha_{r}\right)\right)$. For instance, we will prove certain convergent infinite series expansions (Theorem A(iii) and Remark 2). These results show an interesting new feature of our present situation, because in $[$ Mat1,2] we have obtained similar asymptotic expansions for multiple zeta sums but they are not convergent (Remark 3).

In the second section, we evaluate the special values of our zeta functions and the associated derivatives at $s=0$ in terms of the special values of Hurwitz zeta functions (Theorems D,E) after establishing a certain combinatorial identity. We here only give the formulas for $\zeta(0 ; P, Q)$ and $\zeta^{\prime}(0 ; P, Q)$, but by extending our method, it is possible to show similar formulas for the values at $s=0$ of higher derivatives, and also corresponding formulas at any negative integers.

The evaluation of $\zeta^{\prime}(0 ; P, Q)$ for some special cases has been studied extensively in connection with the regularized determinant of the Laplacian $[\mathrm{V}],[\mathrm{WY}],[\mathrm{QC}],[\mathrm{Ku}]$. The regularized determinant of the Laplacian on the $\left(C^{\infty}\right.$-functions over) $g$-dimensional sphere is given by $\exp \left(-Z_{g}^{\prime}(0)\right)$, where

$$
Z_{g}(s)=\sum_{n=1}^{\infty}\left(\left(\begin{array}{c}
n+g \\
g
\end{array}\right)-\left(\begin{array}{c}
n+g-2 \\
g
\end{array}\right)\right)(n(n+g-1))^{-s}
$$

Hence the evaluation of the regularized determinant in this case is reduced to that for $H_{g, d}^{\prime}(0)$, where

$$
H_{g, d}(s)=\sum_{n=1}^{\infty} \frac{n^{d}}{(n(n+g))^{s}}
$$

Based on [V], an explicit formula for $H_{g, d}^{\prime}(0)$ may be given. See e.g., $[\mathrm{Ku}]$. In the last part of the present paper we show that our Theorem E includes such a result as a special case.

The authors express their gratitude to Professor Yoshio Tanigawa and the referee for useful comments.

\section{Analytic Properties}

\subsection{Meromorphic Extension}

Let $P(x), Q(x)$ be two non-zero polynomials with complex coefficients. Define the associated Dirichlet series $\zeta(s ; P, Q)$ by

$$
\zeta(s ; P, Q):=\sum_{n=1}^{\infty} \frac{P(n)}{Q(n)^{s}} .
$$

Clearly, $\zeta(s ; P, Q)$ is well-defined for $\operatorname{Re}(s)$ sufficiently large, provided that $Q(n) \neq 0$ for all $n=1,2,3, \ldots$ which in this paper we always assume.

In this section, we prove $\zeta(s ; P, Q)$ admits the analytic continuation to the whole complex plane. For this, we study the following more general multiple variable zeta functions.

Let $\alpha_{1}, \ldots, \alpha_{r} \in \mathbf{C} \backslash\{-1,-2, \ldots\}$. Define

$$
\zeta_{r}\left(\left(s_{1}, \ldots, s_{r}\right) ;\left(\alpha_{1}, \ldots, \alpha_{r}\right)\right):=\sum_{n=1}^{\infty}\left(n+\alpha_{1}\right)^{-s_{1}}\left(n+\alpha_{2}\right)^{-s_{2}} \cdots\left(n+\alpha_{r}\right)^{-s_{r}}
$$

This series is well-defined and is clearly convergent absolutely if $\operatorname{Re}\left(s_{1}+s_{2}+\ldots+s_{r}\right)>1$. For our later convenience, we further assume that $\operatorname{Re}_{j}>1, j=1, \ldots, r$. (So $\operatorname{Re}\left(s_{1}+\ldots+s_{r}\right)>r$ indeed.) 
Now to write $\zeta(s ; P, Q)$ in terms of $\zeta_{r}$, first factor $Q(x)$ as $Q(x)=b \prod_{j=1}^{m}\left(x+\beta_{j}\right)$, then expand $P(x)$ in terms of $x+\beta_{1}$ to get $P(x)=: \sum_{i=0}^{n} \tilde{a}_{i}\left(x+\beta_{1}\right)^{i}$ where $\tilde{a}_{n} \neq 0$. Clearly

$$
\zeta(s ; P, Q)=b^{-s} \sum_{i=0}^{n} \tilde{a}_{i} \zeta_{m}\left((s-i, s, \ldots, s) ;\left(\beta_{1}, \beta_{2}, \ldots, \beta_{m}\right)\right) .
$$

Hence our problem is reduced to the analytic continuation of $\zeta_{r}$.

If $r=1$, nothing should be added here. So we may assume that $r \geq 2$. The key is the following classical Mellin-Barnes integral formula

$$
\Gamma(s)(1+\lambda)^{-s}=\frac{1}{2 \pi i} \int_{(C)} \Gamma(s+z) \Gamma(-z) \lambda^{z} d z
$$

where $\Gamma(s)$ denotes the standard gamma function, $s, \lambda \in \mathbf{C}, \operatorname{Re} s>0, \lambda \neq 0,|\arg \lambda|<\pi,-\operatorname{Re} s<C<0$, and the path is the vertical line $\operatorname{Re} z=C$.

Indeed, if $\alpha_{r} \notin \mathbf{R}_{\leq 0}$, from (4) with $s=s_{r}$ and $\lambda=\alpha_{r} / n$, we get

$$
\left(n+\alpha_{1}\right)^{-s_{1}} \ldots\left(n+\alpha_{r}\right)^{-s_{r}}=\frac{1}{2 \pi i} \int_{(C)} \frac{\Gamma\left(s_{r}+z\right) \Gamma(-z)}{\Gamma\left(s_{r}\right)}\left(n+\alpha_{1}\right)^{-s_{1}} \ldots\left(n+\alpha_{r-1}\right)^{-s_{r-1}} \cdot n^{-s_{r}-z} \alpha_{r}^{z} d z
$$

where $-\operatorname{Re} s_{r}<C<0$. Note that

$$
\operatorname{Re}\left(s_{1}+\ldots+s_{r-1}+s_{r}+z\right)>(r-1)+\operatorname{Re}\left(s_{r}\right)+C>r-1 \geq 1 .
$$

Hence

$$
\zeta_{r}\left(\left(s_{1}, \ldots, s_{r}\right) ;\left(\alpha_{1}, \ldots, \alpha_{r}\right)\right)=\frac{1}{2 \pi i} \int_{(C)} \frac{\Gamma\left(s_{r}+z\right) \Gamma(-z)}{\Gamma\left(s_{r}\right)} \cdot \zeta_{r}\left(\left(s_{1}, \ldots, s_{r-1}, s_{r}+z\right) ;\left(\alpha_{1}, \ldots, \alpha_{r-1}, 0\right)\right) \alpha_{r}^{z} d z .
$$

Similarly, from (4) (this time with $s=s_{r-1}$ and $\lambda=\alpha_{r-1} / n$ assuming $\alpha_{r-1} \notin \mathbf{R}_{\leq 0}$ ), we obtain the following relation

$$
\begin{aligned}
& \zeta_{r}\left(\left(s_{1}, \ldots, s_{r-1}, s_{r}\right) ;\left(\alpha_{1}, \ldots, \alpha_{r-1}, 0\right)\right) \\
= & \frac{1}{2 \pi i} \int_{\left(C_{r-1}\right)} \frac{\Gamma\left(s_{r-1}+z_{r-1}\right) \Gamma\left(-z_{r-1}\right)}{\Gamma\left(s_{r-1}\right)} \zeta_{r-1}\left(\left(s_{1}, \ldots, s_{r-2}, s_{r-1}+s_{r}+z_{r-1}\right) ;\left(\alpha_{1}, \ldots, \alpha_{r-2}, 0\right)\right) \alpha_{r-1}^{z_{r-1}} d z_{r-1},
\end{aligned}
$$

where $-\operatorname{Re} s_{r-1}<C_{r-1}-1<C_{r-1}<0$ and $\operatorname{Re} s_{j}>1, j=1, \ldots, r$. This $(7)$ is our basis of the induction procedure.

Remark 1. If $\alpha_{j} \in \mathbf{R}_{\leq 0} \backslash\{-1,-2, \ldots\}$, there exists a positive integer $n_{0}$ such that $n_{0}+\alpha_{j}>0$. Hence (2) becomes

$$
\sum_{n=1}^{n_{0}}\left(n+\alpha_{1}\right)^{-s_{1}} \ldots\left(n+\alpha_{r}\right)^{-s_{r}}+\sum_{n=n_{0}+1}^{\infty}\left(n+\alpha_{1}\right)^{-s_{1}} \ldots\left(n+\alpha_{r}\right)^{-s_{r}},
$$

and the holomorphy of the first sum with respect to $s_{1}, \ldots, s_{r}$ is obvious. Therefore we can reduce the problem to the case $\alpha_{j} \notin \mathbf{R}_{\leq 0}$.

Proposition 1. For any $r \geq 1$,

(i) The $r$-ple zeta function $\zeta_{r}$ defined by (2) with $\alpha_{r}=0$ can be continued meromorphically with respect to $s_{1}, \ldots, s_{r}$ to the whole $\mathbf{C}^{r}$-space, and is holomorphic in $\alpha_{1}, \ldots, \alpha_{r-1}$ if $\alpha_{j} \in \mathbf{C} \backslash \mathbf{R}_{\leq(-1)},(1 \leq j \leq r-1)$;

(ii) The possible singularities of $\zeta_{r}$ are only located on $s_{1}+s_{2}+\ldots+s_{r}=1-k,\left(k \in \mathbf{N}_{0}:=\mathbf{Z}_{\geq 0}\right)$;

(iii) The order estimate

$$
\zeta_{r} \ll \mathcal{F}\left(t_{1}, \ldots, t_{r}\right) \cdot e^{\rho_{1}\left|t_{1}\right|+\rho_{2}\left|t_{2}\right|+\ldots+\rho_{r-1}\left|t_{r-1}\right|}
$$

holds, where $t_{j}=\operatorname{Im} s_{j}$ and $\rho_{j}=\left|\arg \alpha_{j}\right|$. Here, and in what follows, $\mathcal{F}(.$.$) denotes a quantity, not necessarily$ the same at each occurrence, which is of polynomial order in the indicated variables. 
Proof. If $r=1, \zeta_{r}$ is simply the Riemann zeta function. So we are done.

Now assume $r \geq 2$. First consider the case $\alpha_{j} \notin \mathbf{R}_{\leq 0},(1 \leq j \leq r-1)$. Then we may apply (7). Hereafter we assume the validity of Proposition for $\zeta_{r-1}$ and prove the case for $\zeta_{r}$. We write $s_{j}=\sigma_{j}+i t_{j}$ and $z_{j}=x_{j}+i y_{j}(1 \leq j \leq r)$.

By using the order assumption (iii) for $\zeta_{r-1}$, we see that the integrand on the right-hand side of (7) is

$$
\begin{aligned}
& \ll e^{-\frac{\pi}{2}\left|t_{r-1}+y_{r-1}\right|}\left(\left|t_{r-1}+y_{r-1}\right|+1\right)^{\sigma_{r-1}+x_{r-1}-\frac{1}{2}} \\
& \quad \cdot e^{-\frac{\pi}{2}\left|y_{r-1}\right|}\left(\left|y_{r-1}\right|+1\right)^{-x_{r-1}-\frac{1}{2}} \cdot e^{\frac{\pi}{2}\left|t_{r-1}\right|}\left(\left|t_{r-1}\right|+1\right)^{\frac{1}{2}-\sigma_{r-1}} \\
& \quad \cdot \mathcal{F}\left(t_{1}, \ldots, t_{r-2}, t_{r-1}+t_{r}+y_{r-1}\right) \cdot e^{\rho_{1}\left|t_{1}\right|+\ldots+\rho_{r-2}\left|t_{r-2}\right|}\left|\alpha_{r-1}\right|^{x_{r-1}} e^{\rho_{r-1}\left|y_{r-1}\right|} .
\end{aligned}
$$

Since we may assume $\rho_{r-1}<\pi$, we find that the above tends to 0 when $\left|y_{r-1}\right| \rightarrow \infty$. Hence we may shift the path of integration of $(7)$ to $\operatorname{Re} z_{r-1}=M-\varepsilon$, where $M$ is a positive integer. The poles of the integrand are as follows:

(I) poles $-s_{r-1}-k,\left(k \in \mathbf{N}_{0}\right)$, coming from the factor $\Gamma\left(s_{r-1}+z_{r-1}\right)$,

(II) poles $k,\left(k \in \mathbf{N}_{0}\right)$, coming from the factor $\Gamma\left(-z_{r-1}\right)$, and

(III) poles coming from the factor $\zeta_{r-1}$.

By the assumption (ii) for $\zeta_{r-1}$, the poles (III) are

$$
s_{1}+\ldots+s_{r-2}+\left(s_{r-1}+s_{r}+z_{r-1}\right)=1-k, \quad\left(k \in \mathbf{N}_{0}\right),
$$

that is,

$$
z_{r-1}=1-k-\left(s_{1}+\ldots+s_{r}\right)
$$

Since

$$
\operatorname{Re} z_{r-1} \leq 1-\operatorname{Re}\left(s_{1}+\ldots+s_{r}\right)=-\operatorname{Re}\left(s_{1}+\ldots+s_{r-2}\right)-\operatorname{Re} s_{r-1}+\left(1-\operatorname{Re} s_{r}\right)<0+C_{r-1}+0=C_{r-1}
$$

we see that the poles (9) are all located to the left of the line $\operatorname{Re} z_{r-1}=C_{r-1}$. The poles (I) are clearly to the left of the same line, because $-\operatorname{Re} s_{r-1}<C_{r-1}$. Hence the only relevant poles are type (II). Thus, we obtain

$$
\begin{aligned}
& \zeta_{r}\left(\left(s_{1}, \ldots, s_{r}\right) ;\left(\alpha_{1}, \ldots, \alpha_{r-1}, 0\right)\right) \\
= & \sum_{j=0}^{M-1}\left(\begin{array}{c}
-s_{r-1} \\
j
\end{array}\right) \zeta_{r-1}\left(\left(s_{1}, \ldots, s_{r-2}, s_{r-1}+s_{r}+j\right) ;\left(\alpha_{1}, \ldots, \alpha_{r-2}, 0\right)\right) \alpha_{r-1}^{j} \\
+ & \frac{1}{2 \pi i} \int_{(M-\varepsilon)} \frac{\Gamma\left(s_{r-1}+z_{r-1}\right) \Gamma\left(-z_{r-1}\right)}{\Gamma\left(s_{r-1}\right)} \zeta_{r-1}\left(\left(s_{1}, \ldots, s_{r-2}, s_{r-1}+s_{r}+z_{r-1}\right) ;\left(\alpha_{1}, \ldots, \alpha_{r-2}, 0\right)\right) \alpha_{r-1}^{z_{r-1}} d z_{r-1} .
\end{aligned}
$$

Obviously, the poles of the integrand of the above integral, which are (I), (II) and (III) listed above, do not lie on the path of integration if

$$
-\operatorname{Re} s_{r-1}<M-\varepsilon \text { and } 1-\operatorname{Re}\left(s_{1}+\ldots+s_{r}\right)<M-\varepsilon
$$

that is,

$$
\operatorname{Re} s_{r-1}>-M+\varepsilon \text { and } \operatorname{Re}\left(s_{1}+\ldots+s_{r}\right)>1-M+\varepsilon \text {. }
$$

Therefore, since $M$ is arbitrary, (10) gives the continuation of $\zeta_{r}$ to the whole $\mathbf{C}^{r}$-space. The holomorphy with respect to $\alpha_{1}, \ldots, \alpha_{r-1}$ is clear from (10) in case they are $\notin \mathbf{R}_{\leq 0}$. Even if $-1<\alpha_{j} \leq 0$ for some $j$, we can show the meromorphy with respect to $s_{j}$ and the holomorphy with respect to $\alpha_{j}$ by using Remark 1 , because $n+\alpha_{j}>0$ for any positive integer $n$. (If $\alpha_{j}<-1$, we encounter the problem of multi-valuedness of $\log \left(n+\alpha_{j}\right)$.) Moreover, the singularities are only coming from the factor

$$
\zeta_{r-1}\left(\left(s_{1}, \ldots, s_{r-2}, s_{r-1}+s_{r}+j\right) ;\left(\alpha_{1}+\ldots+\alpha_{r-2}, 0\right)\right)
$$


in the first term on the right-hand side of (10). Those singularities are, by the assumption (ii) for $\zeta_{r-1}$,

$$
s_{1}+\ldots+s_{r-2}+\left(s_{r-1}+s_{r}+j\right)=1-k \quad\left(k \in \mathbf{N}_{0}\right)
$$

that is,

$$
s_{1}+\ldots+s_{r}=1-j-k \quad\left(j, k \in \mathbf{N}_{0}\right) .
$$

Therefore we now obtain the assertions (i) and (ii) of Proposition 1 for $\zeta_{r}$.

Next we prove (iii). By using (8), we find that the integral on the right-hand side of (10) is

$$
\begin{aligned}
\ll & \int_{-\infty}^{\infty} \exp \left(-\frac{\pi}{2}\left|t_{r-1}+y_{r-1}\right|-\frac{\pi}{2}\left|y_{r-1}\right|+\frac{\pi}{2}\left|t_{r-1}\right|\right) \cdot \exp \left(\rho_{1}\left|t_{1}\right|+\ldots+\rho_{r-2}\left|t_{r-2}\right|+\rho_{r-1}\left|y_{r-1}\right|\right) \\
& \cdot \mathcal{F}\left(t_{1}, \ldots, t_{r-2}, t_{r-1}, t_{r}, y_{r-1}\right) d y_{r-1} \\
=\exp \left(\rho_{1}\left|t_{1}\right|+\ldots+\rho_{r-2}\left|t_{r-2}\right|+\frac{\pi}{2}\left|t_{r-1}\right|\right) & \\
& \cdot \int_{-\infty}^{\infty} \mathcal{F}\left(t_{1}, \ldots, t_{r}, y_{r-1}\right) \cdot \exp \left(-\frac{\pi}{2}\left|t_{r-1}+y_{r-1}\right|+\left(\rho_{r-1}-\frac{\pi}{2}\right)\left|y_{r-1}\right|\right) d y_{r-1} .
\end{aligned}
$$

Lemma 1. ([Mat2, $\S 5$, Lemma 3]) Let $p, A, B, \alpha, \beta \in \mathbf{R}, A+B<0$. Then

$$
\int_{-\infty}^{\infty}(|y|+1)^{p} \exp (A|y+\alpha|+B|y+\beta|) d y=O\left((|\alpha|+1)^{p+1} e^{B|\alpha-\beta|}+(|\beta|+1)^{p+1} e^{A|\alpha-\beta|}\right)
$$

and the $O$-constant depends only on $p, A$ and $B$.

By this lemma, the integral of the right hand side of (10) is

$$
\begin{aligned}
& \ll \exp \left(\rho_{1}\left|t_{1}\right|+\ldots+\rho_{r-2}\left|t_{r-2}\right|+\frac{\pi}{2}\left|t_{r-1}\right|\right) \\
& \quad \cdot \mathcal{F}\left(t_{1}, \ldots, t_{r}\right) \cdot\left(e^{-\frac{\pi}{2}\left|t_{r-1}\right|}+e^{\left(\rho_{r-1}-\frac{\pi}{2}\right)\left|t_{r-1}\right|}\right) \\
& \ll \mathcal{F}\left(t_{1}, \ldots, t_{r}\right) \cdot \exp \left(\rho_{1}\left|t_{1}\right|+\ldots+\rho_{r-2}\left|t_{r-2}\right|+\rho_{r-1}\left|t_{r-1}\right|\right) .
\end{aligned}
$$

On the other hand, by using the assumption (iii) for $\zeta_{r-1}$ again, we find that the first term on the right-hand side of $(10)$ is

$$
\mathcal{F}\left(t_{1}, \ldots, t_{r}\right) \cdot e^{\rho_{1}\left|t_{1}\right|+\ldots+\rho_{r-2}\left|t_{r-2}\right|} .
$$

Therefore, we obtain

$$
\begin{aligned}
& \zeta_{r}\left(\left(s_{1}, \ldots, s_{r}\right) ;\left(\alpha_{1}, \ldots, \alpha_{r-1}, 0\right)\right) \\
\ll & \mathcal{F}\left(t_{1}, \ldots, t_{r}\right) \cdot \exp \left(\rho_{1}\left|t_{1}\right|+\ldots+\rho_{r-2}\left|t_{r-2}\right|+\rho_{r-1}\left|t_{r-1}\right|\right),
\end{aligned}
$$

which is the assertion (iii) of Proposition 1 for $\zeta_{r}$ (with $\alpha_{r}=0$ ). The proof of Proposition 1 is now complete.

Moreover, from (10) we obtain the asymptotic expansion

$$
\begin{aligned}
& \zeta_{r}\left(\left(s_{1}, \ldots, s_{r}\right) ;\left(\alpha_{1}, \ldots, \alpha_{r-1}, 0\right)\right) \\
= & \sum_{j=0}^{M-1}\left(\begin{array}{c}
-s_{r-1} \\
j
\end{array}\right) \zeta_{r-1}\left(\left(s_{1}, \ldots, s_{r-2}, s_{r-1}+s_{r}+j\right) ;\left(\alpha_{1}, \ldots, \alpha_{r-2}, 0\right)\right) \alpha_{r-1}^{j}+O\left(\left|\alpha_{r-1}\right|^{M-\varepsilon}\right)
\end{aligned}
$$

with respect to $\left|\alpha_{r-1}\right|$ when $\left|\alpha_{r-1}\right| \rightarrow 0$. Similarly, we may deduce the asymptotic expansion with respect to $\left|\alpha_{r-1}\right|$ when $\left|\alpha_{r-1}\right| \rightarrow \infty$ by shifting the path of integration to the left.

Proposition 1 gives sufficient analytic information on function $\zeta_{r}\left(\left(s_{1}, \ldots, s_{r}\right) ;\left(\alpha_{1}, \ldots, \alpha_{r-1}, 0\right)\right)$. Now finally, combining Proposition 1 with the formula (6), we deduce analytic information on general zeta functions $\zeta_{r}\left(\left(s_{1}, \ldots, s_{r}\right) ;\left(\alpha_{1}, \ldots, \alpha_{r}\right)\right)$. 
We shift the path of integration on the right-hand side of (6) to $\operatorname{Re} z=M-\varepsilon$. By using the estimate Proposition 1(iii), we find that the integrand of (6) is

$$
\begin{array}{r}
\ll e^{-\frac{\pi}{2}\left|t_{r}+y\right|}\left(\left|t_{r}+y\right|+1\right)^{\sigma_{r}+x-\frac{1}{2}} e^{-\frac{\pi}{2}|y|}(|y|+1)^{-x-\frac{1}{2}} e^{\frac{\pi}{2}\left|t_{r}\right|}\left(\left|t_{r}\right|+1\right)^{\frac{1}{2}-\sigma_{r}} \\
\cdot \mathcal{F}\left(t_{1}, \ldots, t_{r-1}, t_{r}+y\right) \cdot \exp \left(\rho_{1}\left|t_{1}\right|+\ldots+\rho_{r-1}\left|t_{r-1}\right|\right)\left|\alpha_{r}\right|^{x} e^{\rho_{r}|y|}
\end{array}
$$

$\left(\rho_{j}=\left|\arg \alpha_{j}\right|, 1 \leq j \leq r\right)$ which tends to zero when $|y| \rightarrow \infty$ if $\rho_{r}<\pi$, and we have already known (Remark 1) that we may assume this inequality $\rho_{r}<\pi$. Hence the shifting (indicated above) is possible. From Proposition 1(ii) we see that the poles of $\zeta_{r}\left(\left(s_{1}, \ldots, s_{r-1}, s_{r}+z\right) ;\left(\alpha_{1}, \ldots, \alpha_{r-1}, 0\right)\right)$ are

$$
s_{1}+\ldots+s_{r-1}+\left(s_{r}+z\right)=1-k,
$$

that is

$$
z=1-\left(s_{1}+\ldots+s_{r}\right)-k, \quad\left(k \in \mathbf{N}_{0}\right) .
$$

When $\operatorname{Re}_{j}>1,(1 \leq j \leq r)$, we have

$$
\begin{aligned}
\operatorname{Re} z \leq & 1-\operatorname{Re}\left(s_{1}+\ldots+s_{r}\right)=1-\operatorname{Re}\left(s_{1}+\ldots+s_{r-1}\right)-\operatorname{Re} s_{r} \\
& <-\operatorname{Re} s_{r} \quad(\text { if } r \geq 2) \\
& <C,
\end{aligned}
$$

that is, these poles are on the left of the original path $\operatorname{Re} z=C$. Thus the only relevant poles are $z=$ $0,1,2, \ldots, M-1$. Counting the residues of those poles, we obtain

$$
\begin{aligned}
& \zeta_{r}\left(\left(s_{1}, \ldots, s_{r}\right) ;\left(\alpha_{1}, \ldots, \alpha_{r}\right)\right) \\
= & \sum_{k=0}^{M-1}\left(\begin{array}{c}
-s_{r} \\
k
\end{array}\right) \zeta_{r}\left(\left(s_{1}, \ldots, s_{r-1}, s_{r}+k\right) ;\left(\alpha_{1}, \ldots, \alpha_{r-1}, 0\right)\right) \alpha_{r}^{k} \\
& +\frac{1}{2 \pi i} \int_{(M-\varepsilon)} \frac{\Gamma\left(s_{r}+z\right) \Gamma(-z)}{\Gamma\left(s_{r}\right)} \cdot \zeta_{r}\left(\left(s_{1}, \ldots, s_{r-1}, s_{r}+z\right) ;\left(\alpha_{1}, \ldots, \alpha_{r-1}, 0\right)\right) \alpha_{r}^{z} d z .
\end{aligned}
$$

The poles of the integrand are

$$
z=-s_{r}-k, z=k, z=1-\left(s_{1}+\ldots+s_{r}\right)-k, \quad\left(k \in \mathbf{N}_{0}\right),
$$

which do not lie on the path $\operatorname{Re} z=M-\varepsilon$ if

$$
-\operatorname{Re} s_{r}<M-\varepsilon \quad \text { and } \quad 1-\operatorname{Re}\left(s_{1}+\ldots+s_{r}\right)<M-\varepsilon .
$$

Therefore the integral on the right-hand side of (12) is holomorphic (as a function in $s_{1}, \ldots, s_{r}$ ) in the region

$$
\operatorname{Re} s_{r}>-M+\varepsilon \quad \text { and } \quad \operatorname{Re}\left(s_{1}+\ldots+s_{r}\right)>1-M+\varepsilon .
$$

Since $M$ is arbitrary, now (12) implies the meromorphic continuation of $\zeta_{r}\left(\left(s_{1}, \ldots, s_{r}\right) ;\left(\alpha_{1}, \ldots, \alpha_{r}\right)\right)$ to the whole $\mathbf{C}^{r}$-space. All singularities are coming from the first term

$$
\sum_{k=0}^{M-1}\left(\begin{array}{c}
-s_{r} \\
k
\end{array}\right) \zeta_{r}\left(\left(s_{1}, \ldots, s_{r-1}, s_{r}+k\right) ;\left(\alpha_{1}, \ldots, \alpha_{r-1}, 0\right)\right) \alpha_{r}^{k}
$$

Hence, by using Proposition 1(ii), we find that $\zeta_{r}\left(\left(s_{1}, \ldots, s_{r}\right) ;\left(\alpha_{1}, \ldots, \alpha_{r}\right)\right)$ is holomorphic except for the possible singularities at

$$
s_{1}+\ldots+s_{r}=1-k \quad\left(k \in \mathbf{N}_{0}\right) .
$$

Therefore we now obtain the first and the second assertions of the following 
Theorem A. For any $r \geq 1$,

(i) The r-ple zeta function $\zeta_{r}$ defined by (2) can be continued meromorphically with respect to $s_{1}, \ldots, s_{r}$ to the whole $\mathbf{C}^{r}$-space, and is holomorphic in $\alpha_{1}, \ldots, \alpha_{r}$ if $\alpha_{j} \in \mathbf{C} \backslash \mathbf{R}_{\leq(-1)}(1 \leq j \leq r)$;

(ii) The possible singularities of $\zeta_{r}$ are only located at $s_{1}+s_{2}+\ldots+s_{r}=1-k,\left(k \in \mathbf{N}_{0}:=\mathbf{Z}_{\geq 0}\right)$;

(iii) If $\left|\alpha_{r}\right|<1$, then

$$
\zeta_{r}\left(\left(s_{1}, \ldots, s_{r}\right) ;\left(\alpha_{1}, \ldots, \alpha_{r}\right)\right)=\sum_{k=0}^{\infty}\left(\begin{array}{c}
-s_{r} \\
k
\end{array}\right) \zeta_{r}\left(\left(s_{1}, \ldots, s_{r-1}, s_{r}+k\right) ;\left(\alpha_{1}, \ldots, \alpha_{r-1}, 0\right)\right) \alpha_{r}^{k} .
$$

Proof of Theorem A(iii). This is just the Taylor expansion of $\zeta_{r}$ with respect to $\alpha_{r}$. The radius of convergence is 1 , because the assertion (i) implies that the singularity of $\alpha_{r}$ nearest to the origin is $\alpha_{r}=-1$.

Obviously, from Theorem A, we obtain the following

Theorem B. Let $P(x), Q(x)$ be polynomials with complex coefficients. Assume that all roots $-\beta_{1}, \ldots,-\beta_{m}$ of $Q(x)$ are not in $\mathbf{R}_{\geq 1}$. Define the Dirichlet series $\zeta(s ; P, Q)$ associated to $P$ and $Q$ by

$$
\zeta(s ; P, Q):=\sum_{k=1}^{\infty} \frac{P(k)}{Q(k)^{s}} .
$$

Then, $\zeta(s ; P, Q)$ can be meromorphically extended to the whole complex s-plane, and is holomorphic in $\beta_{1}, \ldots, \beta_{m}$.

Now set

$$
I_{M}=\frac{1}{2 \pi i} \int_{(M-\varepsilon)} \frac{\Gamma\left(s_{r}+z\right) \Gamma(-z)}{\Gamma\left(s_{r}\right)} \zeta_{r}\left(\left(s_{1}, \ldots, s_{r-1}, s_{r}+z\right) ;\left(\alpha_{1}, \ldots, \alpha_{r-1}, 0\right)\right) \alpha_{r}^{z} d z .
$$

From (12) and Theorem A(iii), we obtain

Lemma 2. With the same notation as above, $\lim _{M \rightarrow \infty} I_{M}=0$ if $\left|\alpha_{r}\right|<1$.

In view of Remark 3 below, it is of interest to give a direct proof of this fact. The following argument is valid if $\left|\arg \alpha_{r}\right|<\pi$.

Using $\Gamma(-z)=-\frac{\pi}{\Gamma(1+z) \sin (\pi z)}$ and Stirling's formula $\Gamma(z)=\sqrt{2 \pi} e^{-z} z^{z-\frac{1}{2}}\left(1+O\left(\frac{1}{|z|}\right)\right.$, we have

$$
I_{M}=\frac{i e}{2 e^{s_{r}} \Gamma\left(s_{r}\right)} \int_{(M-\varepsilon)} \frac{\left(z\left(1+\frac{s_{r}}{z}\right)\right)^{s_{r}+z-\frac{1}{2}}}{\left(z\left(1+\frac{1}{z}\right)\right)^{z+\frac{1}{2}} \sin (\pi z)}\left(1+O\left(\frac{1}{|z|}\right)\right) \zeta_{r}\left(\left(s_{1}, \ldots, s_{r-1}, s_{r}+z\right) ;\left(\alpha_{1}, \ldots, \alpha_{r-1}, 0\right)\right) \alpha_{r}^{z} d z
$$

Clearly,

and

$$
\left(1+\frac{s_{r}}{z}\right)^{s_{r}+z-\frac{1}{2}}=\exp \left(s_{r}+O\left(\frac{1}{|z|}\right)\right)=e^{s_{r}}\left(1+O\left(\frac{1}{|z|}\right)\right)
$$

$$
\left(1+\frac{1}{z}\right)^{z+\frac{1}{2}}=e \cdot\left(1+O\left(\frac{1}{|z|}\right)\right) .
$$

Hence,

$$
I_{M}=\frac{i e}{2 e^{s_{r}} \Gamma\left(s_{r}\right)} \cdot e^{s_{r}-1} \cdot \int_{(M-\varepsilon)} \frac{z^{s_{r}-1}}{\sin (\pi z)}\left(1+O\left(\frac{1}{|z|}\right)\right) \times \zeta_{r}\left(\left(s_{1}, \ldots, s_{r-1}, s_{r}+z\right) ;\left(\alpha_{1}, \ldots, \alpha_{r-1}, 0\right)\right) \alpha_{r}^{z} d z .
$$

Now write $\mu=M-\varepsilon, z=\mu+i y$. Note that, since $\mu \notin \mathbf{Z},\left|e^{\pi i(\mu+i y)}-e^{-\pi i(\mu+i y)}\right|>>e^{|y|}$ for any $y \in \mathbf{R}$. Thus, writing $\sin (\pi z)=\frac{1}{2 i}\left(e^{\pi i z}-e^{-\pi i z}\right)$, we have

$$
I_{M} \ll \frac{1}{\left|\Gamma\left(s_{r}\right)\right|} \int_{-\infty}^{\infty} \frac{\left|(\mu+i y)^{s_{r}-1}\right|}{e^{\pi|y|}}\left(1+O\left(\frac{1}{1+|y|}\right)\right)\left|\zeta_{r}\left(\left(s_{1}, \ldots, s_{r-1}, s_{r}+z\right) ;\left(\alpha_{1}, \ldots, \alpha_{r-1}, 0\right)\right)\right| \cdot\left|\alpha_{r}\right|^{\mu} e^{\rho_{r}|y|} d y .
$$


Write $s_{r}=\sigma_{r}+i t_{r}$, then

$$
\left|(\mu+i y)^{s_{r}-1}\right| \leq|\mu+i y|^{\sigma_{r}-1} e^{\frac{\pi}{2}\left|t_{r}\right|}
$$

Thus, from $1+O\left(\frac{1}{1+|y|}\right) \ll 1$, we have

$$
I_{M} \ll \frac{e^{\frac{\pi}{2}\left|t_{r}\right|}}{\left|\Gamma\left(s_{r}\right)\right|} \int_{-\infty}^{\infty} \frac{\left|(\mu+i y)^{\sigma_{r}-1}\right|}{e^{\pi|y|}}\left|\zeta_{r}\left(\left(s_{1}, \ldots, s_{r-1}, s_{r}+z\right) ;\left(\alpha_{1}, \ldots, \alpha_{r-1}, 0\right)\right)\right| \cdot\left|\alpha_{r}\right|^{\mu} e^{\rho_{r}|y|} d y .
$$

Thus, by the fact that $\zeta_{r}\left(\left(s_{1}, \ldots, s_{r-1}, s_{r}+z\right) ;\left(\alpha_{1}, \ldots, \alpha_{r-1}, 0\right)\right)$ is absolutely convergent if $\operatorname{Re}(z)=\mu=M-\varepsilon$ is sufficiently large, we get

$$
I_{M} \ll \int_{-\infty}^{\infty} \frac{\left|(\mu+i y)^{\sigma_{r}-1}\right|}{e^{\pi|y|}} \cdot\left|\alpha_{r}\right|^{\mu} e^{\rho_{r}|y|} d y .
$$

But if $|y| \leq \mu$ (resp. $|y|>\mu)$, then $|\mu+i y| \sim \mu$ (resp. $|\mu+i y| \sim|y|)$, hence we have

$$
\begin{aligned}
I_{M} & \ll \int_{-\mu}^{\mu} \frac{\mu^{\sigma_{r}-1}}{e^{\pi|y|}} \cdot\left|\alpha_{r}\right|^{\mu} e^{\rho_{r}|y|} d y+\int_{|y|>\mu} \frac{|y|^{\sigma_{r}-1}}{e^{\pi|y|}} \cdot\left|\alpha_{r}\right|^{\mu} e^{\rho_{r}|y|} d y \\
& \leq \mu^{\sigma_{r}-1}\left|\alpha_{r}\right|^{\mu} \int_{-\infty}^{\infty} \frac{d y}{e^{\left(\pi-r h o_{r}\right)|y|}}+\left|\alpha_{r}\right|^{\mu} \int_{|y|>\mu} \frac{|y|^{\sigma_{r}-1}}{e^{\left(\pi-\rho_{r}\right)|y|}} d y .
\end{aligned}
$$

If $\rho_{r}=\left|\arg \alpha_{r}\right|<\pi$, we have

$$
I_{M} \ll \mu^{\sigma_{r}-1}\left|\alpha_{r}\right|^{\mu}+\left|\alpha_{r}\right|^{\mu}
$$

where $\ll$ depends on $\sigma_{r}$. Therefore $\lim _{M \rightarrow \infty} I_{M}=0$ if $\left|\alpha_{r}\right|<1$.

Remark 2. Similarly to Theorem A(iii), we can prove that, if $\left|\alpha_{r-1}\right|<1$,

$$
\begin{aligned}
& \zeta_{r}\left(\left(s_{1}, \ldots, s_{r}\right) ;\left(\alpha_{1}, \ldots, \alpha_{r-1}, 0\right)\right) \\
= & \sum_{j=0}^{\infty}\left(\begin{array}{c}
-s_{r-1} \\
j
\end{array}\right) \zeta_{r-1}\left(\left(s_{1}, \ldots, s_{r-2}, s_{r-1}+s_{r}+j\right) ;\left(\alpha_{1}, \ldots, \alpha_{r-2}, 0\right)\right) \alpha_{r-1}^{j} .
\end{aligned}
$$

Remark 3. In [Mat 1,2], we have encountered asymptotic expansions which are similar to (11), (12) but each term in the expansions includes an additional factor like $\zeta(-k, b)$, where $\zeta(s, b)$ stands for the Hurwitz zeta function. By using the formula (2.17.3) of [T] it is easily seen that $|\zeta(-k, b)| \sim(2 e \pi)^{-k} k^{k+\frac{1}{2}}$, which implies that the expansions in $[$ Mat 1,2] are not convergent.

\subsection{Regularity at non-positive integers}

Now we consider the situation at non-positive integers of $\zeta_{r}\left((s-k, s, \ldots, s) ;\left(\alpha_{1}, \ldots, \alpha_{r}\right)\right)$. By (12), it is enough to consider the case $\alpha_{r}=0$, that is, $\zeta_{r}\left((s-k, s, \ldots, s) ;\left(\alpha_{1}, \ldots, \alpha_{r-1}, 0\right)\right)$. Let $n \in \mathbf{N}$ and $h \in \mathbf{N}_{0}$. We study the following somewhat more general form: $\zeta_{r}\left((s-k, s, \ldots, s, n s+h) ;\left(\alpha_{1}, \ldots, \alpha_{r-1}, 0\right)\right)$.

First we discuss the case $r=2$. By (10) we have

$$
\begin{aligned}
& \zeta_{2}\left((s-k, n s+h) ;\left(\alpha_{1}, 0\right)\right) \\
= & \sum_{j=0}^{M-1}\left(\begin{array}{c}
-s+k \\
j
\end{array}\right) \zeta((n+1) s-k+h+j) \alpha_{1}^{j}+(\text { integral term }) .
\end{aligned}
$$

Since the integral term is holomorphic, the poles are coming only from the factors $\zeta((n+1) s-k+h+j)$. Hence

Lemma 3. All poles of $\zeta_{2}\left((s-k, n s+h) ;\left(\alpha_{1}, 0\right)\right)$ are at most of order 1 . 
The poles of $\zeta((n+1) s-k+h+j)$ is at $(n+1) s-k+h+j=1$, that is

$$
s=\frac{k-h-j+1}{n+1} .
$$

On the other hand, we have

$$
\left(\begin{array}{cl}
-s+k \\
j
\end{array}\right)= \begin{cases}1, & \text { if } j=0 \\
\frac{1}{j !}(-s+k)(-s+k-1) \ldots(-s+k-j+1), & \text { if } j \geq 1\end{cases}
$$

and the zeros of the latter are at

$$
s=k, k-1, \ldots, k-j+1 .
$$

When $j=0$, the pole $s=\frac{k-h+1}{n+1}$ is really a pole of $\zeta_{2}\left((s-k, n s+h) ;\left(\alpha_{1}, 0\right)\right)$. When $j \geq 1$, the pole $\frac{k-h-j+1}{n+1}$ is cancelled by a zero in the list of (14) if and only if there exists an

$$
l \in \mathbf{N}_{0}, 0 \leq l \leq j-1, \frac{k-h-j+1}{n+1}=k-l
$$

These conditions are valid if and only if $\frac{k-h-j+1}{n+1} \in \mathbf{Z}$ and $j \geq k+1+\frac{h}{n}$. That is,

$$
\frac{k-h-j+1}{n+1} \in \mathbf{Z} \quad \text { and } \quad \frac{k-h-j+1}{n+1} \leq \frac{k-h-\left(k+1+\frac{h}{n}\right)+1}{n+1}=-\frac{h}{n} .
$$

Summarizing the above argument, we now obtain

Lemma 4. The set of all poles of $\zeta_{2}\left((s-k, n s+h) ;\left(\alpha_{1}, 0\right)\right)$ is

$$
\left\{\frac{k-h-j+1}{n+1} ; j \geq 0\right\} \backslash\left\{\text { integers } \leq-\frac{h}{n}\right\},
$$

and poles are all simple.

Note that, from $(13)$, the residue of $\zeta_{2}((s-k, n s+h) ;(\alpha, 0))$ at $s=\frac{k-h-j+1}{n+1}$ is

$$
=\frac{\alpha_{1}^{j}}{n+1}\left(\begin{array}{c}
k-\frac{k-h-j+1}{n+1} \\
j
\end{array}\right)
$$

Next we consider the case $r=3$. By (10), we have

$$
\zeta_{3}\left((s-k, s, n s+h) ;\left(\alpha_{1}, \alpha_{2}, 0\right)\right)=\sum_{j=0}^{M-1}\left(\begin{array}{c}
-s \\
j
\end{array}\right) \zeta_{2}\left((s-k,(n+1) s+h+j) ;\left(\alpha_{1}, 0\right)\right) \alpha_{2}^{j}+(\text { integral term }) .
$$

Using Lemma 4, we have that $\zeta_{2}\left((s-k,(n+1) s+h+j) ;\left(\alpha_{1}, 0\right)\right)$ has a pole at $s=\frac{k-(h+j)-j^{\prime}+1}{(n+1)+1}, j^{\prime} \geq 0$ and the residue there is by (16)

$$
\frac{\alpha_{1}^{j^{\prime}}}{(n+1)+1}\left(\begin{array}{c}
k-\frac{k-(h+j)-j^{\prime}+1}{(n+1)+1} \\
j^{\prime}
\end{array}\right) .
$$

Let $j+j^{\prime}=l$. The same $s=\frac{k-h-l+1}{n+2}, l \geq 0$ appears from the pairs $\left(j, j^{\prime}\right)=(0, l),(1, l-1), \ldots,(l, 0)$. (We assume $M$ is sufficiently large so $M-1 \geq l$.) Therefore, the residue at $s=\frac{k-h-l+1}{n+2}$ of $\zeta_{3}((s-k, s, n s+$ $\left.h) ;\left(\alpha_{1}, \alpha_{2}, 0\right)\right)$ is equal to

$$
\sum_{j=0}^{l}\left(\begin{array}{c}
-\frac{k-h-l+1}{n+2} \\
j
\end{array}\right) \cdot \frac{\alpha_{1}^{j^{\prime}}}{(n+1)+1}\left(\begin{array}{c}
k-\frac{k-h-l+1}{(n+1)+1} \\
j^{\prime}
\end{array}\right) \alpha_{2}^{j}=\frac{1}{n+2} \sum_{j=0}^{l}\left(\begin{array}{c}
-\frac{k-h-l+1}{n+2} \\
j
\end{array}\right)\left(\begin{array}{c}
k-\frac{k-h-l+1}{n+2} \\
l-j
\end{array}\right) \alpha_{1}^{l-j} \alpha_{2}^{j} .
$$




$$
\frac{k-h-l+1}{n+2}=-m \in \mathbf{Z}, m \geq 0,
$$

then $\left(\frac{-\frac{k-h-l+1}{n+2}}{j}\right)=0$ for $j \geq m+1$ and $\left({ }^{k-\frac{k-h-l+1}{n+2}} \frac{l-j}{l+j}\right)=0$ for $j \leq l-k-m-1$. Therefore we can conclude that if

$$
m \leq l-k-m-1,
$$

then the right hand side of (17) is zero, so actually $\zeta_{3}\left((s-k, s, n s+h) ;\left(\alpha_{1}, \alpha_{2}, 0\right)\right)$ is holomorphic at $s=-m$. The condition (19) is equivalent to $l \geq k+2 m+1$, hence from (18) we have

$$
-m(n+2)=k-h-l+1 \leq k-h-(k+2 m+1)+1,
$$

i.e., $m \geq \frac{h}{n}$. This argument implies that the negative integers $-m$ with $m \geq \frac{h}{n}$ is not singular.

Now we study the general case. The above results suggest that the function

$$
\zeta_{r}\left((s-k, s, \ldots, s, n s+h) ;\left(\alpha_{1}, \ldots, \alpha_{r-1}, 0\right)\right)
$$

is holomprphic at $s=-m$, which is an integer and $\leq-\frac{h}{n}$. We prove this fact by induction. Assume that the above claim is true for $\zeta_{r-1}$. Recall that we have

$$
\begin{aligned}
& \zeta_{r}\left((s-k, s, \ldots, s, n s+h) ;\left(\alpha_{1}, \ldots, \alpha_{r-1}, 0\right)\right) \\
= & \sum_{j=0}^{M-1}\left(\begin{array}{c}
-s \\
j
\end{array}\right) \zeta_{r-1}\left((s-k, s, \ldots, s,(n+1) s+h+j) ;\left(\alpha_{1}, \ldots, \alpha_{r-2}, 0\right)\right) \alpha_{r-1}^{j}+(\text { holomorphic term })
\end{aligned}
$$

where in particular the binomial coefficients are $\left(\begin{array}{c}-s \\ j\end{array}\right)$ instead of $\left(\begin{array}{c}-s+k \\ j\end{array}\right)$ as $r \geq 3$. First, it is easy to see that Lemma 5. All poles of $\zeta_{r}\left((s-k, s, \ldots, s, n s+h) ;\left(\alpha_{1}, \ldots, \alpha_{r-1}, 0\right)\right),\left(n \in \mathbf{N}, h \in \mathbf{N}_{0}\right)$ are at most of order 1.

Proof. We already know this fact for $r=2$. (See e.g., Lemma 3.) Hence, by using (20), the general case immediately follows by induction.

The induction hypothesis says that $\zeta_{r-1}\left((s-k, s, \ldots, s,(n+1) s+h+j) ;\left(\alpha_{1}, \ldots, \alpha_{r-2}, 0\right)\right)$ is holomorphic at $s=-m, m \in \mathbf{Z}, m \geq \frac{h+j}{n+1}$. On the other hand, by Lemma 5 we see that

$$
\left(\begin{array}{c}
-s \\
j
\end{array}\right) \zeta_{r-1}\left((s-k, s, \ldots, s,(n+1) s+h+j) ;\left(\alpha_{1}, \ldots, \alpha_{r-2}, 0\right)\right)
$$

is not singular at $s=0,-1,-2,-3, \ldots,-(j-1)$. Hence we find that if

$$
\frac{h+j}{n+1} \leq j
$$

then the term (21) is non-singular for any non-positive integer. The condition (22) is equivalent to $h+j \leq$ $(n+1) j$, hence $j \geq \frac{h}{n}$. This implies that the terms on the right-hand side of (20) with $j \geq \frac{h}{n}$ are non-singular at non-positive integers.

Consider the terms $(21)$ with $0 \leq j<\frac{h}{n}$. We have already shown that these terms are holomorphic at $s=-m \in \mathbf{Z}, m \geq \frac{h+j}{n+1}$. Hence if $s=-m, m \in \mathbf{Z}_{\geq 0}$ is singular, then $0 \leq m<\frac{h+j}{n+1}$. Since $j<\frac{h}{n}$ we find

$$
m<\frac{h+\frac{h}{n}}{n+1}=\frac{h}{n}
$$

Therefore if $s=-m, m \in \mathbf{Z}_{\geq 0}$, is a pole of the right hand side of (20), it is necessary that $m<\frac{h}{n}$. In other words, $s=-m, m \in \mathbf{Z}_{\geq 0}, m \geq \frac{h}{n}$ is not a singular point of (20) as desired. We have proved the following 
Proposition 2. The function $\zeta_{r}\left((s-k, s, \ldots, s, n s+h) ;\left(\alpha_{1}, \ldots, \alpha_{r-1}, 0\right)\right)$ is holomorphic at any integer $s=-m \leq-\frac{h}{n}$. In particular, $\zeta_{r}\left((s-k, s, \ldots, s) ;\left(\alpha_{1}, \ldots, \alpha_{r-1}, 0\right)\right)$ is holomorphic at any non-positive integers.

The same conclusion holds for $\zeta_{r}\left((s-k, s, \ldots, s, n s+h) ;\left(\alpha_{1}, \ldots, \alpha_{r-1}, \alpha_{r}\right)\right)$, which can be easily checked by using (12). As a direct consequence, we obtain the following

Theorem C. For any two polynomials $P(x)$ and $Q(x)$, the associated zeta function $\zeta(s ; P, Q)$ is holomorphic at any non-positive integers.

\section{Special Values}

\subsection{The Value $\zeta(0 ; P, Q)$}

Let $P(x), Q(x), \zeta(s ; P, Q)$ be as in the previous section. Denote $n:=\operatorname{deg} P, m:=\operatorname{deg} Q$ and in this section we write $P(x)=\sum_{i=0}^{n} a_{i} x^{i}$ and $Q(x)=b \prod_{j=1}^{m}\left(x+\beta_{j}\right)$. Assume that $\beta_{j} \notin \mathbf{R}_{\leq(-1)}, 1 \leq j \leq m$. Then by the results in Section $1, \zeta(s ; P, Q)$ is holomorphic at $s=0$. Hence it makes sense to talk about $\zeta(0 ; P, Q)$ and $\zeta^{\prime}(0 ; P, Q)$. In this section, we evaluate those values.

Lemma 6. Assume $\left|\beta_{j}\right|<1(1 \leq j \leq m)$. Then we have

$$
\zeta(0 ; P, Q)=\sum_{i=0}^{n} a_{i} \zeta(-i)+\frac{1}{m} \sum_{j=1}^{m} \sum_{i=0}^{n} \frac{a_{i}}{i+1}\left(-\beta_{j}\right)^{i+1}
$$

Proof. By a direct calculation using $\frac{1}{\left(k+\beta_{j}\right)^{s}}=\frac{1}{k^{s}} \sum_{l_{j}=0}^{\infty}\left(\begin{array}{c}-s \\ l_{j}\end{array}\right)\left(\frac{\beta_{j}}{k}\right)^{l_{j}}$, we have

$$
\begin{aligned}
\zeta(s ; P, Q)= & \sum_{k=1}^{\infty} \frac{P(k)}{b^{s}\left(k+\beta_{1}\right)^{s} \ldots\left(k+\beta_{m}\right)^{s}} \\
= & \sum_{l_{1}, \ldots, l_{m}=0}^{\infty} b^{-s}\left(\beta_{1}\right)^{l_{1}} \ldots\left(\beta_{m}\right)^{l_{m}}\left(\begin{array}{c}
-s \\
l_{1}
\end{array}\right) \ldots\left(\begin{array}{c}
-s \\
l_{m}
\end{array}\right) \sum_{k=1}^{\infty} \sum_{i=0}^{n} a_{i} k^{i} k^{-m s-l_{1}-\ldots-l_{m}} \\
= & \sum_{i=0}^{n} a_{i} \sum_{l_{1}, \ldots, l_{m}=0}^{\infty} b^{-s}\left(\beta_{1}\right)^{l_{1}} \ldots\left(\beta_{m}\right)^{l_{m}}\left(\begin{array}{c}
-s \\
l_{1}
\end{array}\right) \ldots\left(\begin{array}{c}
-s \\
l_{m}
\end{array}\right) \zeta\left(m s+l_{1}+\ldots+l_{m}-i\right) \\
= & \sum_{i=0}^{n} a_{i} b^{-s}\left[\zeta(m s-i)+\sum_{j=1}^{m} \sum_{l_{j}=1}^{\infty}\left(\beta_{j}\right)^{l_{j}}\left(\begin{array}{c}
-s \\
l_{j}
\end{array}\right) \zeta\left(m s+l_{j}-i\right)\right. \\
& \left.+\sum_{\#\left\{j ; l_{j} \neq 0\right\} \geq 2}\left(\beta_{1}\right)^{l_{1}} \ldots\left(\beta_{m}\right)^{l_{m}}\left(\begin{array}{c}
-s \\
l_{1}
\end{array}\right) \ldots\left(\begin{array}{c}
-s \\
l_{m}
\end{array}\right) \zeta\left(m s+l_{1}+\ldots+l_{m}-i\right)\right] .
\end{aligned}
$$

Therefore, noting $\zeta(s)=\frac{1}{s-1}+c_{0}+c_{1}(s-1)+\ldots$, and $\left(\begin{array}{l}0 \\ l\end{array}\right)=0$ if $l \neq 0$, we get

$$
\begin{aligned}
& \zeta(0 ; P, Q)=\sum_{i=0}^{n} a_{i}\left[\zeta(-i)+\left.\sum_{j=1}^{m} \sum_{l_{j}=1}^{\infty}\left(\beta_{j}\right)^{l_{j}}\left(\begin{array}{c}
-s \\
l_{j}
\end{array}\right) \zeta\left(m s+l_{j}-i\right)\right|_{s=0}\right] \\
& =\sum_{i=0}^{n} a_{i}\left[\zeta(-i)+\left.\sum_{j=1}^{m}\left(\beta_{j}\right)^{i+1}\left(\begin{array}{c}
-s \\
i+1
\end{array}\right) \zeta(m s+1)\right|_{s=0}\right] \\
& =\sum_{i=0}^{n} a_{i} \zeta(-i)+\left.\sum_{i=0}^{n} a_{i} \sum_{j=1}^{m}\left(\beta_{j}\right)^{i+1} \frac{(-s)(-s-1) \ldots(-s-(i+1)+1)}{(i+1) !} \zeta(m s+1)\right|_{s=0}
\end{aligned}
$$




$$
\begin{aligned}
& =\sum_{i=0}^{n} a_{i} \zeta(-i)+\sum_{i=0}^{n} a_{i} \sum_{j=1}^{m}\left(\beta_{j}\right)^{i+1} \frac{(-1)^{i+1}}{i+1} \cdot \frac{1}{m} \\
& =\sum_{i=0}^{n} a_{i} \zeta(-i)+\frac{1}{m} \sum_{j=1}^{m} \sum_{i=0}^{n} \frac{a_{i}}{i+1}\left(-\beta_{j}\right)^{i+1} .
\end{aligned}
$$

This completes the proof of Lemma 6 .

In view of Theorem $\mathrm{B}$, the left-hand side of the formula in the statement of Lemma 6 is holomorphic in $\beta_{j} \in \mathbf{C} \backslash \mathbf{R}_{\leq(-1)}(1 \leq j \leq m)$. Hence by analytic continuation with respect to $\beta_{1}, \ldots, \beta_{m}$, we have the following

Theorem D. Let $P(x), Q(x)$ be as above. Assume that $\beta_{j} \notin \mathbf{R}_{\leq(-1)}, 1 \leq j \leq m$ and define the Dirichlet series $\zeta(s ; P, Q):=\sum_{n=1}^{\infty} \frac{P(n)}{Q(n)^{s}}$. Then,

$$
\zeta(0 ; P, Q)=\sum_{i=0}^{n} a_{i} \zeta(-i)+\frac{1}{m} \sum_{j=1}^{m} \sum_{i=0}^{n} \frac{a_{i}}{i+1}\left(-\beta_{j}\right)^{i+1} .
$$

\subsection{The Value $\zeta^{\prime}(0 ; P, Q)$}

In this subsection, we evaluate $\zeta^{\prime}(0 ; P, Q)$. We first introduce the following auxiliary functions.

$$
\begin{aligned}
A(s):= & \sum_{j=1}^{m} \sum_{k=1}^{\infty} \frac{P(k)}{Q(k)^{s}} \log \left(k+\beta_{j}\right), \\
B(s):= & b^{-s} \sum_{i=0}^{n} a_{i} \sum_{j=1}^{m} \sum_{u=0}^{i}\left(\begin{array}{c}
i \\
u
\end{array}\right)\left(-\beta_{j}\right)^{i-u} \sum_{l_{1}, \ldots, \hat{l}_{j}, \ldots, l_{m}=0}^{\infty}\left(\beta_{1}-\beta_{j}\right)^{l_{1}} \ldots\left(\beta_{m}-\beta_{j}\right)^{l_{m}}\left(\begin{array}{c}
-s \\
l_{1}
\end{array}\right) \ldots\left(\begin{array}{c}
-s \\
l_{m}
\end{array}\right) \\
& \times \sum_{k=1}^{\infty}\left(k+\beta_{j}\right)^{-m s-l_{1}-\ldots-l_{m}+u} \log \left(k+\beta_{j}\right) .
\end{aligned}
$$

Here $\hat{l}_{j}$ means that $l_{j}$ is omitted from the summation.

Lemma 7. With the same notation as above, under the assumption $\left|\beta_{j}\right|<1 / 3(1 \leq j \leq m)$, we have,

(i) $\zeta^{\prime}(0 ; P, Q)=-\zeta(0 ; P, Q) \log b-A(0)$;

(ii) $A(0)=B(0)$ and hence $\zeta^{\prime}(0 ; P, Q)=-\zeta(0 ; P, Q) \log b-B(0)$.

Proof. The proof of (i) is given by a direct calculation. As for (ii), we first note that the assumption $\left|\beta_{j}\right|<1 / 3(1 \leq j \leq m)$ implies

$$
\left|\frac{\beta_{p}-\beta_{j}}{k+\beta_{j}}\right|<1 \quad(1 \leq j \leq m, 1 \leq p \leq m)
$$

for any positive integer $k$. Therefore

$$
\left(k+\beta_{p}\right)^{-s}=\left(k+\beta_{j}\right)^{-s}\left(1+\frac{\beta_{p}-\beta_{j}}{k+\beta_{j}}\right)^{-s}=\left(k+\beta_{j}\right)^{-s} \sum_{l_{p}=0}^{\infty}\left(\begin{array}{c}
-s \\
l_{p}
\end{array}\right)\left(\frac{\beta_{p}-\beta_{j}}{k+\beta_{j}}\right)^{l_{p}}
$$

and similarly

$$
k^{i}=\left(k+\beta_{j}\right)^{i} \sum_{u=0}^{i}\left(\begin{array}{l}
i \\
u
\end{array}\right)\left(\frac{-\beta_{j}}{k+\beta_{j}}\right)^{i-u} .
$$

Using these formulas we obtain $A(0)=B(0)$, hence (ii) follows. 

define

Next we consider $B(0)$. For this, we again introduce the following auxiliary functions. First, for $\beta_{j}$,

$$
\zeta_{j}(s)=\sum_{k=1}^{\infty} \frac{1}{\left(k+\beta_{j}\right)^{s}},
$$

and denote its derivatives by $\zeta_{j}^{\prime}$. Note that $\zeta_{j}(s)=\zeta\left(s, 1+\beta_{j}\right)$, where the right-hand side denotes the standard Hurwitz zeta-function. In the sequel we sometimes write $\zeta_{j}^{\prime}(m s+c)$, which means $\left.(d / d w) \zeta_{j}(w)\right|_{w=m s+c}$. We set

$$
\begin{aligned}
& B^{1}(s):=b^{-s} \sum_{i=0}^{n} a_{i} \sum_{j=1}^{m} \sum_{u=0}^{i}\left(\begin{array}{l}
i \\
u
\end{array}\right)\left(-\beta_{j}\right)^{i-u} \zeta_{j}^{\prime}(m s-u), \\
& B^{2}(s):=b^{-s} \sum_{i=0}^{n} a_{i} \sum_{j=1}^{m} \sum_{u=0}^{i}\left(\begin{array}{l}
i \\
u
\end{array}\right)\left(-\beta_{j}\right)^{i-u} \sum_{p \neq j, p=1}^{m} \sum_{l_{p}=1}^{\infty}\left(\beta_{p}-\beta_{j}\right)^{l_{p}}\left(\begin{array}{c}
-s \\
l_{p}
\end{array}\right) \zeta_{j}^{\prime}\left(m s+l_{p}-u\right) \text {, } \\
& B^{3}(s)=b^{-s} \sum_{i=0}^{n} a_{i} \sum_{j=1}^{m} \sum_{u=0}^{i}\left(\begin{array}{l}
i \\
u
\end{array}\right)\left(-\beta_{j}\right)^{i-u} \\
& \times \sum_{j \neq p, j \neq q, p \neq q, p, q=1}^{m} \sum_{l_{p}, l_{q}=1}^{\infty}\left(\beta_{p}-\beta_{j}\right)^{l_{p}}\left(\beta_{q}-\beta_{j}\right)^{l_{q}}\left(\begin{array}{c}
-s \\
l_{p}
\end{array}\right)\left(\begin{array}{c}
-s \\
l_{q}
\end{array}\right) \zeta_{j}^{\prime}\left(m s+l_{p}+l_{q}-u\right), \\
& B^{4}(s):=b^{-s} \sum_{i=0}^{n} a_{i} \sum_{j=1}^{m} \sum_{u=0}^{i}\left(\begin{array}{l}
i \\
u
\end{array}\right)\left(-\beta_{j}\right)^{i-u} \sum_{\#\left\{p \neq j ; l_{p} \neq 0\right\} \geq 3} \sum_{l_{1}, \ldots, l_{j}, \ldots, l_{m}=0}^{\infty}\left(\beta_{1}-\beta_{j}\right)^{l_{1}} \ldots\left(\beta_{m}-\beta_{j}\right)^{l_{m}} \\
& \times\left(\begin{array}{c}
-s \\
l_{1}
\end{array}\right) \ldots\left(\begin{array}{c}
-s \\
l_{m}
\end{array}\right) \zeta_{j}^{\prime}\left(m s+l_{1}+\ldots+\hat{l}_{j}+\ldots+l_{m}-u\right) .
\end{aligned}
$$

Since the innermost sum in the definition of $B(s)$ is $-\zeta_{j}^{\prime}\left(m s+l_{1}+\ldots+\hat{l}_{j}+\ldots+l_{m}-u\right)$, by a direct calculation, we have the following

Lemma 8. With the same notation as above, under the assumption $\left|\beta_{j}\right|<1 / 3(1 \leq j \leq m)$, we have,

$$
B(0)=-B^{1}(0)-B^{2}(0)-B^{3}(0)-B^{4}(0)
$$

In particular,

$$
\zeta^{\prime}(0 ; P, Q)=-\zeta(0 ; P, Q) \log b+B^{1}(0)+B^{2}(0)+B^{3}(0)+B^{4}(0)
$$

Lemma 9. With the same notation as above, under the assumption $\left|\beta_{j}\right|<1 / 3(1 \leq j \leq m)$, we have,

(i) $B^{1}(0)=\sum_{j=1}^{m} \sum_{i=0}^{n} a_{i} \sum_{u=0}^{i}\left(\begin{array}{c}i \\ u\end{array}\right)\left(-\beta_{j}\right)^{i-u} \zeta_{j}^{\prime}(-u)$;

(ii) $B^{2}(0)=-\frac{1}{m^{2}} \sum_{i=0}^{n} a_{i} \sum_{j=1}^{m} \sum_{u=0}^{i}\left(\begin{array}{c}i \\ u\end{array}\right)\left(-\beta_{j}\right)^{i-u} \sum_{p=1}^{m}\left(\beta_{j}-\beta_{p}\right)^{u+1} \frac{1}{u+1} \sum_{a=1}^{u} \frac{1}{a}$;

(iii) $B^{3}(0)=-\frac{1}{m^{2}} \sum_{j=1}^{m} \sum_{i=0}^{n} a_{i} \sum_{u=0}^{i}\left(\begin{array}{c}i \\ u\end{array}\right)\left(-\beta_{j}\right)^{i-u} \sum_{p \neq q, p, q=1}^{m} \cdot \sum_{l_{p}, l_{q} \geq 1, l_{p}+l_{q}=1+u} \frac{\left(-\beta_{p}+\beta_{j}\right)^{l_{p}}}{l_{p}} \frac{\left(-\beta_{q}+\beta_{j}\right)^{l_{q}}}{l_{q}}$;

(iv) $B^{4}(0)=0$.

Proof. First, from the definition, (i) follows immediately.

Note that $\zeta_{j}(s)=\frac{1}{s-1}+c_{0}+c_{1}(s-1)+\ldots$ (see e.g., $\left.[\mathrm{I}]\right)$. Thus by $\left(\begin{array}{l}0 \\ l\end{array}\right)=0$ if $l \neq 0$, from the definition, $B^{4}(0)=0$, which gives (iv). 
As for (iii), we have

$$
\begin{aligned}
& B^{3}(0)=b^{-s} \sum_{i=0}^{n} a_{i} \sum_{j=1}^{m} \sum_{u=0}^{i}\left(\begin{array}{l}
i \\
u
\end{array}\right)\left(-\beta_{j}\right)^{i-u} \\
& \times \sum_{j \neq p, j \neq q, p \neq q, p, q=1, l_{p}+l_{q}-u=1}^{m}\left(\beta_{p}-\beta_{j}\right)^{l_{p}}\left(\beta_{q}-\beta_{j}\right)^{l_{q}} \\
& \left.\frac{(-s)(-s-1) \ldots\left(-s-l_{p}+1\right)}{l_{p} !} \cdot \frac{(-s)(-s-1) \ldots\left(-s-l_{q}+1\right)}{l_{q} !} \cdot \frac{-1}{m^{2} s^{2}}\right|_{s=0} \\
& =\sum_{i=0}^{n} a_{i} \sum_{j=1}^{m} \sum_{u=0}^{i}\left(\begin{array}{l}
i \\
u
\end{array}\right)\left(-\beta_{j}\right)^{i-u} \sum_{j \neq p, j \neq q, p \neq q, p, q=1}^{m} \\
& \times \sum_{l_{p}, l_{q} \geq 1, l_{p}+l_{q}=1+u}\left(\beta_{p}-\beta_{j}\right)^{l_{p}}\left(\beta_{q}-\beta_{j}\right)^{l_{q}} \frac{(-1)^{l_{p}-1}}{l_{p}} \cdot \frac{(-1)^{l_{q}-1}}{l_{q}} \cdot \frac{-1}{m^{2}} \\
& =-\frac{1}{m^{2}} \sum_{j=1}^{m} \sum_{i=0}^{n} a_{i} \sum_{u=0}^{i}\left(\begin{array}{l}
i \\
u
\end{array}\right)\left(-\beta_{j}\right)^{i-u} \sum_{p \neq q, p, q=1}^{m} \cdot \sum_{l_{p}, l_{q} \geq 1, l_{p}+l_{q}=1+u} \frac{\left(-\beta_{p}+\beta_{j}\right)^{l_{p}}}{l_{p}} \frac{\left(-\beta_{q}+\beta_{j}\right)^{l_{q}}}{l_{q}} .
\end{aligned}
$$

This gives (iii).

Finally, let us consider $B^{2}(s)$. For this, we need the following

Sublemma. With the same notation as above, we have

$$
\sum_{j=1}^{m} \sum_{p=1}^{m}\left(\sum_{u=0}^{i}\left(\begin{array}{l}
i \\
u
\end{array}\right) \frac{1}{u+1}\left(-\beta_{j}\right)^{i-u}\left(\beta_{j}-\beta_{p}\right)^{u+1}\right)=0 .
$$

Proof. Clearly,

$$
\begin{aligned}
& \sum_{j=1}^{m} \sum_{p=1}^{m}\left(\sum_{u=0}^{i}\left(\begin{array}{c}
i \\
u
\end{array}\right) \frac{1}{u+1}\left(-\beta_{j}\right)^{i-u}\left(\beta_{j}-\beta_{p}\right)^{u+1}\right) \\
= & \frac{1}{i+1} \sum_{j, p=1}^{m} \sum_{u=0}^{i}\left(\begin{array}{c}
i+1 \\
u+1
\end{array}\right)\left(-\beta_{j}\right)^{(i+1)-(u+1)}\left(\beta_{j}-\beta_{p}\right)^{u+1} \\
= & \frac{1}{i+1} \sum_{j, p=1}^{m} \sum_{k=1}^{i+1}\left(\begin{array}{c}
i+1 \\
k
\end{array}\right)\left(-\beta_{j}\right)^{(i+1)-k}\left(\beta_{j}-\beta_{p}\right)^{k} \\
= & \frac{1}{i+1} \sum_{j, p=1}^{m}\left(\sum_{k=0}^{i+1}\left(\begin{array}{c}
i+1 \\
k
\end{array}\right)\left(-\beta_{j}\right)^{(i+1)-k}\left(\beta_{j}-\beta_{p}\right)^{k}-\left(-\beta_{j}\right)^{i+1}\right) \\
= & \frac{1}{i+1} \sum_{j, p=1}^{m}\left(\left(-\beta_{j}+\beta_{j}-\beta_{p}\right)^{i+1}-\left(-\beta_{j}\right)^{i+1}\right) \\
= & 0 .
\end{aligned}
$$

This completes the proof of the sublemma.

Now let us come back to the proof of Lemma 9(ii). By the definition,

$$
\begin{aligned}
B^{2}(0) & =\left.b^{-s} \sum_{i=0}^{n} a_{i} \sum_{j=1}^{m} \sum_{u=0}^{i}\left(\begin{array}{l}
i \\
u
\end{array}\right)\left(-\beta_{j}\right)^{i-u} \sum_{p=1}^{m} \sum_{l_{p}=1}^{\infty}\left(\beta_{p}-\beta_{j}\right)^{l_{p}}\left(\begin{array}{c}
-s \\
l_{p}
\end{array}\right) \zeta_{j}^{\prime}\left(m s+l_{p}-u\right)\right|_{s=0} \\
& =\left.b^{-s} \sum_{i=0}^{n} a_{i} \sum_{j=1}^{m} \sum_{u=0}^{i}\left(\begin{array}{l}
i \\
u
\end{array}\right)\left(-\beta_{j}\right)^{i-u} \sum_{p=1}^{m}\left(\beta_{p}-\beta_{j}\right)^{u+1}\left(\begin{array}{c}
-s \\
u+1
\end{array}\right) \zeta_{j}^{\prime}(m s+1)\right|_{s=0}
\end{aligned}
$$




$$
\begin{aligned}
& =b^{-s} \sum_{i=0}^{n} a_{i} \sum_{j=1}^{m} \sum_{u=0}^{i}\left(\begin{array}{c}
i \\
u
\end{array}\right)\left(-\beta_{j}\right)^{i-u} \\
& \times\left.\sum_{p=1}^{m}\left(\beta_{p}-\beta_{j}\right)^{u+1} \frac{(-s)(-s-1) \ldots(-s-(u+1)+1)}{(u+1) !}\left(-\frac{1}{m^{2}} \cdot \frac{1}{s^{2}}+\text { holomorphic terms }\right)\right|_{s=0} \\
& =-\left.\frac{1}{m^{2}} b^{-s} \sum_{i=0}^{n} a_{i} \sum_{j=1}^{m} \sum_{u=0}^{i}\left(\begin{array}{l}
i \\
u
\end{array}\right)\left(-\beta_{j}\right)^{i-u} \sum_{p=1}^{m}\left(\beta_{j}-\beta_{p}\right)^{u+1} \frac{(s+1) \ldots(s+u)}{(u+1) !}\left(\frac{1}{s}\right)\right|_{s=0} \\
& =-\left.\frac{1}{m^{2}} b^{-s} \sum_{i=0}^{n} a_{i} \sum_{j=1}^{m} \sum_{u=0}^{i}\left(\begin{array}{l}
i \\
u
\end{array}\right)\left(-\beta_{j}\right)^{i-u} \sum_{p=1}^{m}\left(\beta_{j}-\beta_{p}\right)^{u+1} \frac{u !+\sum_{a=1}^{u} u ! / a \cdot s}{(u+1) !}\left(\frac{1}{s}\right)\right|_{s=0} \\
& =-\left.\frac{1}{m^{2}} b^{-s} \sum_{i=0}^{n} a_{i} \sum_{j=1}^{m} \sum_{u=0}^{i}\left(\begin{array}{l}
i \\
u
\end{array}\right)\left(-\beta_{j}\right)^{i-u} \sum_{p=1}^{m}\left(\beta_{j}-\beta_{p}\right)^{u+1}\left(\frac{1}{u+1}+\frac{\sum_{a=1}^{u} u ! / a \cdot s}{(u+1) !}\right)\left(\frac{1}{s}\right)\right|_{s=0} \\
& =-\frac{1}{m^{2}} \sum_{i=0}^{n} a_{i} \sum_{j=1}^{m} \sum_{u=0}^{i}\left(\begin{array}{c}
i \\
u
\end{array}\right)\left(-\beta_{j}\right)^{i-u} \sum_{p=1}^{m}\left(\beta_{j}-\beta_{p}\right)^{u+1} \frac{1}{u+1} \sum_{a=1}^{u} \frac{1}{a}
\end{aligned}
$$

provided that

$$
\sum_{i=0}^{n} a_{i} \sum_{j=1}^{m} \sum_{u=0}^{i}\left(\begin{array}{l}
i \\
u
\end{array}\right)\left(-\beta_{j}\right)^{i-u} \sum_{p=1}^{m}\left(\beta_{j}-\beta_{p}\right)^{u+1} \frac{1}{u+1}=0 .
$$

But by the sublemma above,

$$
\sum_{u=0}^{i}\left(\begin{array}{l}
i \\
u
\end{array}\right) \frac{1}{u+1} \sum_{j=1}^{m} \sum_{p=1}^{m}\left(-\beta_{j}\right)^{i-u}\left(\beta_{j}-\beta_{p}\right)^{u+1}=0 .
$$

This then completes the proof of (ii) and hence Lemma 9.

By analytic continuation, we may remove the assumption $\left|\beta_{j}\right|<1 / 3(1 \leq j \leq m)$. Therefore, we have the following

Theorem E. Let $P(x)=\sum_{i=0}^{n} a_{i} x^{i}, Q(x)=b \prod_{j=1}^{m}\left(x+\beta_{j}\right)$ be polynomials with complex coefficients. Assume that $\beta_{j} \notin \mathbf{R}_{\leq(-1)}, 1 \leq j \leq m$. Define the Dirichlet series $\zeta(s ; P, Q):=\sum_{n=1}^{\infty} \frac{P(n)}{Q(n)^{s}}$. Then,

$$
\begin{aligned}
\zeta^{\prime}(0 ; P, Q)= & -\zeta(0 ; P, Q) \log b+\sum_{j=1}^{m} \sum_{i=0}^{n} a_{i} \sum_{u=0}^{i}\left(\begin{array}{c}
i \\
u
\end{array}\right)\left(-\beta_{j}\right)^{i-u} \zeta_{j}^{\prime}(-u) \\
& -\frac{1}{m^{2}} \sum_{j=1}^{m} \sum_{i=0}^{n} a_{i} \sum_{u=0}^{i}\left(\begin{array}{l}
i \\
u
\end{array}\right)\left(-\beta_{j}\right)^{i-u} \sum_{p \neq q, p, q=1}^{m} \sum_{l_{p}, l_{q} \geq 1, l_{p}+l_{q}=1+u} \frac{\left(-\beta_{p}+\beta_{j}\right)^{l_{p}}}{l_{p}} \frac{\left(-\beta_{q}+\beta_{j}\right)^{l_{q}}}{l_{q}} \\
& -\frac{1}{m^{2}} \sum_{j=1}^{m} \sum_{i=0}^{n} a_{i} \sum_{u=0}^{i}\left(\begin{array}{l}
i \\
u
\end{array}\right)\left(-\beta_{j}\right)^{i-u} \sum_{p=1}^{m}\left(\beta_{j}-\beta_{p}\right)^{u+1} \frac{1}{u+1} \sum_{a=1}^{u} \frac{1}{a} .
\end{aligned}
$$

Here $\zeta_{j}(s):=\sum_{k=1}^{\infty} \frac{1}{\left(k+\beta_{j}\right)^{s}}=\zeta\left(s, 1+\beta_{j}\right)$ denotes the standard Hurwitz zeta function associated to $\beta_{j}$.

\subsection{Determinant of the Laplacian}

Concerning the zeta-function $H_{g, d}(s)$ defined by (5) in the Introduction, Kumagai [Ku, Lemma 3] proves that

$$
\begin{aligned}
H_{g, d}^{\prime}(0)= & \sum_{k=1}^{g}(k-g)^{d} \log k-\frac{1}{2^{d}} \cdot \frac{(-g)^{d+1}}{d+1} \sum_{1 \leq l \leq d ; l: \text { odd }}\left(\begin{array}{l}
d+1 \\
l+1
\end{array}\right) \sum_{1 \leq j \leq l, j \text { :odd }} \frac{1}{j} \\
& +\zeta^{\prime}(-d)+(-g)^{d} \sum_{r=0}^{d}\left(\begin{array}{l}
d \\
r
\end{array}\right) \frac{\zeta^{\prime}(-r)}{(-g)^{r}} .
\end{aligned}
$$


On the other hand, Theorem E with $P(x)=x^{d}, Q(x)=x(x+g)$ (and hence $\beta_{1}=0, \beta_{2}=g$ ) implies

$$
H_{g, d}^{\prime}(0)=\zeta^{\prime}(-d)+\sum_{u=0}^{d}\left(\begin{array}{l}
d \\
u
\end{array}\right)(-g)^{d-u} \zeta^{\prime}(-u, 1+g)-\frac{1}{4} \cdot(-g)^{d+1}\left(\frac{1}{d+1} \sum_{j=1}^{d} \frac{1}{j}+\sum_{u=1}^{d}\left(\begin{array}{l}
d \\
u
\end{array}\right) \frac{(-1)^{u+1}}{u+1} \sum_{j=1}^{u} \frac{1}{j}\right) \text {. }
$$

Substituting the standard formula

$$
\zeta^{\prime}(-u, 1+g)=\zeta^{\prime}(-u)+\sum_{k=2}^{g} k^{u} \log k,
$$

we find that the right-hand side of (2) is

$$
\begin{aligned}
&=\zeta^{\prime}(-d)+(-g)^{d} \sum_{u=0}^{d}\left(\begin{array}{l}
d \\
u
\end{array}\right)(-g)^{-u} \zeta^{\prime}(-u)+\sum_{u=0}^{d}\left(\begin{array}{l}
d \\
u
\end{array}\right)(-g)^{d-u} \sum_{k=2}^{g} k^{u} \log k \\
&-\frac{1}{4} \cdot \frac{(-g)^{d+1}}{d+1}\left(\sum_{j=1}^{d} \frac{1}{j}+\sum_{u=1}^{d}\left(\begin{array}{l}
d+1 \\
u+1
\end{array}\right)(-1)^{u+1} \sum_{j=1}^{u} \frac{1}{j}\right) .
\end{aligned}
$$

We will show that the above coincides with the right-hand side of (1). Since the third term of the above is equal to $\sum_{k=2}^{g}(k-g)^{d} \log k$, what we have to check is that

$$
\sum_{1 \leq l \leq d, l: \text { odd }}\left(\begin{array}{l}
d+1 \\
l+1
\end{array}\right) \sum_{1 \leq j \leq l, j: \text { odd }} \frac{1}{j}=2^{d-2}\left(\sum_{j=1}^{d} \frac{1}{j}+\sum_{u=1}^{d}(-1)^{u+1}\left(\begin{array}{l}
d+1 \\
u+1
\end{array}\right) \sum_{j=1}^{u} \frac{1}{j}\right) .
$$

Moreover, noting

$$
\left(\begin{array}{l}
d+1 \\
u+1
\end{array}\right)=\left(\begin{array}{c}
d \\
u+1
\end{array}\right)+\left(\begin{array}{l}
d \\
u
\end{array}\right) \quad \text { for } u<d
$$

we have

$$
\begin{aligned}
& \sum_{u=1}^{d}(-1)^{u+1}\left(\begin{array}{l}
d+1 \\
u+1
\end{array}\right) \sum_{j=1}^{u} \frac{1}{j} \\
= & \sum_{u=1}^{d-1}(-1)^{u+1}\left(\begin{array}{c}
d \\
u+1
\end{array}\right) \sum_{j=1}^{u} \frac{1}{j}-\sum_{u=1}^{d-1}(-1)^{u}\left(\begin{array}{l}
d \\
u
\end{array}\right) \sum_{j=1}^{u} \frac{1}{j}+(-1)^{d+1} \sum_{j=1}^{d} \frac{1}{j} \\
= & \sum_{u=2}^{d}(-1)^{u}\left(\begin{array}{l}
d \\
u
\end{array}\right) \sum_{j=1}^{u-1} \frac{1}{j}-\sum_{u=1}^{d-1}(-1)^{u}\left(\begin{array}{l}
d \\
u
\end{array}\right)\left(\sum_{j=1}^{u-1} \frac{1}{j}+\frac{1}{u}\right)+(-1)^{d+1} \sum_{j=1}^{d} \frac{1}{j} \\
= & d-\sum_{j=2}^{d}(-1)^{j}\left(\begin{array}{l}
d \\
j
\end{array}\right) \frac{1}{j} .
\end{aligned}
$$

Therefore, it suffices to prove the following

Lemma 10. The following identity holds:

$$
\sum_{1 \leq l \leq d, l: \text { odd }}\left(\begin{array}{l}
d+1 \\
l+1
\end{array}\right) \sum_{1 \leq j \leq l, j \text { :odd }} \frac{1}{j}=2^{d-2}\left(\sum_{j=1}^{d} \frac{1}{j}+d-\sum_{j=2}^{d}(-1)^{j}\left(\begin{array}{l}
d \\
j
\end{array}\right) \frac{1}{j}\right) .
$$

Before proving this Lemma, let us consider the left-hand side first. For this, let

$$
\begin{aligned}
S(l) & :=\sum_{1 \leq j \leq l, j \text { :odd }} \frac{1}{j}, \\
I(d) & :=\sum_{1 \leq l \leq d, l \text { :odd }}\left(\begin{array}{l}
d+1 \\
l+1
\end{array}\right) S(l), \\
J(d) & :=\sum_{0 \leq l \leq d-1, l \text { :even }}\left(\begin{array}{l}
d+1 \\
l+1
\end{array}\right) S(l+1) .
\end{aligned}
$$


Then the left-hand side of $(5)$ is just $I(d)$, and we have

$$
\begin{gathered}
I(d)=I(d-1)+J(d-1)+\delta(d) S(d), \\
J(d)=J(d-1)+I(d-1)+(2 \delta(d)-1) S(d)+\frac{2^{d}}{d+1}-\frac{1-\delta(d)}{d+1},
\end{gathered}
$$

where $\delta(d)=1$ or 0 according as $d$ is odd or even. In fact, (6) comes directly from (4). As for (7), by definition and (4), we have

$$
\begin{aligned}
J(d) & =J(d-1)+\delta(d) S(d)+\sum_{0 \leq l \leq d-1, l \text { :even }}\left(\begin{array}{l}
d \\
l
\end{array}\right) S(l+1) \\
& =J(d-1)+\delta(d) S(d)+1+\sum_{0 \leq k \leq d-2, k \text { :odd }}\left(\begin{array}{c}
d \\
k+1
\end{array}\right) S(k+2) \\
& =(J(d-1)+\delta(d) S(d)+1)+I(d-1)-(1-\delta(d)) S(d-1)+\sum_{1 \leq k \leq d-2, k \text { odd }}\left(\begin{array}{c}
d \\
k+1
\end{array}\right) \frac{1}{k+2} .
\end{aligned}
$$

Here in the last step we use the fact that $S(k+2)=S(k)+\frac{1}{k+2}$ for $k$ odd. But $\left(\begin{array}{c}d \\ k+1\end{array}\right) \frac{1}{k+2}=\frac{1}{d+1}\left(\begin{array}{c}d+1 \\ k+2\end{array}\right)$. Hence to complete the proof of $(7)$, it suffices to show that

$$
\sum_{1 \leq k \leq d-2, k: \text { odd }}\left(\begin{array}{l}
d+1 \\
k+2
\end{array}\right)=2^{d}-(d+1)-(1-\delta(d))
$$

which may be deduced from

$$
\sum_{1 \leq k \leq d-2}\left(\begin{array}{l}
d+1 \\
k+2
\end{array}\right)=\sum_{h=3}^{d}\left(\begin{array}{c}
d+1 \\
h
\end{array}\right)=2^{d+1}-d-3-\frac{1}{2}(d+1)(d+2)
$$

and the similar identity

$$
\sum_{1 \leq k \leq d-2}(-1)^{k}\left(\begin{array}{l}
d+1 \\
k+2
\end{array}\right)=d-\frac{1}{2}(d+1)(d+2)-(-1)^{d+1} .
$$

Now, from (6) and (7), we obtain the following recursion formula

$$
I(d+1)-I(d)=I(d)+\delta(d+1) S(d+1)+(\delta(d)-1) S(d)+\frac{2^{d}}{d+1}-\frac{1-\delta(d)}{d+1} .
$$

Easily,

$$
\delta(d+1) S(d+1)+(\delta(d)-1) S(d)-\frac{1-\delta(d)}{d+1}=0 .
$$

Hence, we arrive at the relation

$$
I(d+1)=2 I(d)+\frac{2^{d}}{d+1} .
$$

Proof of Lemma 10. Induction on $d$. Clearly if $d=1,2,3,(5)$ holds. Assume now that (5) is valid for $d \geq 3$, then from the induction hypothesis and (8), we have

$$
I(d+1)=2^{d-1}\left(\sum_{j=1}^{d+1} \frac{1}{j}+\frac{1}{d+1}+d-\sum_{j=2}^{d}(-1)^{j}\left(\begin{array}{l}
d \\
j
\end{array}\right) \frac{1}{j}\right) .
$$


Thus it suffices to show that

$$
\frac{1}{d+1}-\sum_{j=2}^{d}(-1)^{j}\left(\begin{array}{l}
d \\
j
\end{array}\right) \frac{1}{j}=1-\sum_{j=2}^{d+1}(-1)^{j}\left(\begin{array}{c}
d+1 \\
j
\end{array}\right) \frac{1}{j}
$$

Noting $\left(\begin{array}{c}d \\ j-1\end{array}\right) \frac{1}{j}=\frac{1}{d+1}\left(\begin{array}{c}d+1 \\ j\end{array}\right)$ as before, we have

$$
\begin{aligned}
& \sum_{j=2}^{d+1}(-1)^{j}\left(\begin{array}{c}
d+1 \\
j
\end{array}\right) \frac{1}{j} \\
= & \sum_{j=2}^{d}(-1)^{j}\left(\left(\begin{array}{l}
d \\
j
\end{array}\right)+\left(\begin{array}{c}
d \\
j-1
\end{array}\right)\right) \frac{1}{j}+(-1)^{d+1} \frac{1}{d+1} \\
= & \sum_{j=2}^{d}(-1)^{j}\left(\begin{array}{l}
d \\
j
\end{array}\right) \frac{1}{j}+\frac{1}{d+1} \sum_{j=2}^{d}(-1)^{j}\left(\begin{array}{c}
d+1 \\
j
\end{array}\right)+(-1)^{d+1} \frac{1}{d+1} \\
= & \sum_{j=2}^{d}(-1)^{j}\left(\begin{array}{l}
d \\
j
\end{array}\right) \frac{1}{j}+\frac{1}{d+1}\left(d-(-1)^{d+1}\right)+(-1)^{d+1} \frac{1}{d+1} \\
= & \sum_{j=2}^{d}(-1)^{j}\left(\begin{array}{l}
d \\
j
\end{array}\right) \frac{1}{j}+1-\frac{1}{d+1}
\end{aligned}
$$

which is equivalent to (9). This completes the proof of Lemma 10, and hence establish the equivalence of (1) and (2). 


\section{REFERENCES}

[CN1] P. Cassou-Noguès, Prolongement de certaines séries de Dirichlet, Amer. J. Math. 105 (1983), 13-58.

[CN2] P. Cassou-Noguès, Valeurs aux entiers négatifs des series de Dirichlet associées a un polynôme I, II, III, J.Number Theory 14 (1982), 32-64; Amer. J. Math. 106 (1984), 255-299; ibid. 109 (1987), 71-89.

[CN3] P. Cassou-Noguès, Séries de Dirichlet et intégrales associées à un polynôme à deux indèterminèes, J.Number Theory 23 (1986), 1-54.

[E1] M. Eie, On a Dirichlet series associated with a polynomial, Proc. Amer. Math. Soc. 110 (1990), 583-590.

[E2] M. Eie, The special values at negative integers of Dirichlet series associated with polynomials of several variables, Proc. Amer. Math. Soc. 119 (1993), 51-61.

[I] A. Ivić, The Riemann zeta-function, Wiley (1985)

[K1] M. Katsurada, An application of Mellin-Barnes' type integrals to the mean square of Lerch zetafunctions, Collect. Math. 48 (1997), 137-153.

[K2] M. Katsurada, An application of Mellin-Barnes type integrals to the mean square of $L$-functions, Liet. Mat. Rink. 38 (1998), 98-112.

[Ku] H. Kumagai, The determinant of the Laplacian on the n-sphere, Acta Arith. 91(1999), 199-208.

[L1] B. Lichtin, Generalized Dirichlet series and b-functions, Compositio Math. 65 (1988), 81-120. (Erratum: ibid. 72 (1989), 237-239.)

[L2] B. Lichtin, Poles of Dirichlet series and D-modules, in "Théorie des Nombres/Number Theory" (Proc. Intern. Number Theory Conf., Laval, 1987), J.-M. De Coninck and C.Levesque (eds.), Walter de Gruyter, 1989, pp.579-594.

[L3] B. Lichtin, On the moderate growth of generalized Dirichlet series for hypoelliptic polynomials, Compositio Math. 80 (1991), 337-354.

[L4] B. Lichtin, The asymptotics of a lattice point problem associated to a finite number of polynomials I, Duke Math. J. 63 (1991), 139-192.

[L5] B. Lichtin, Volumes and lattice points - proof of a conjecture of L.Ehrenpreis, in "Singularities" (Lille, 1991), J.-P. Brasselet (ed.), London Math. Soc. Lecture Note Ser. Vol. 201, Cambridge Univ. Press, 1994, pp.211-250.

[L6] B. Lichtin, The asymptotics of a lattice point problem associated to a finite number of polynomials II, Duke Math. J. 77 (1995), 699-751.

[L7] B. Lichtin, Asymptotics determined by pairs of additive polynomials, Compositio Math. 107 (1997), 233-267.

[Mah] K. Mahler, Über einen Satz von Mellin, Math. Ann. 100 (1928), 384-398.

[Mat1] K. Matsumoto, Asymptotic expansions of double zeta-functions of Barnes, of Shintani, and Eisenstein series, preprint.

[Mat2] K. Matsumoto, The analytic continuation and the asymptotic behavior of multiple zeta-functions I, preprint.

[Me1] H. Mellin, Eine Formel für den Logarithmus transcendenter Funktionen von endlichem Geschlecht, Acta Soc. Sci. Fenn. 29, no.4 (1900).

[Me2] H. Mellin, Die Dirichlet'schen Reihen, die zahlentheoretischen Funktionen und die unendlichen Produkte von endlichem Geschlecht, Acta Math. 28 (1904), 37-64.

[P] M. Peter, Dirichlet series associated with polynomials, Acta Arith. 84 (1998), 245-278.

[QC] J.R. Quine and J. Choi, Zeta regularized products and functional determinants on spheres, Rocky Mountain J. Math. 26(1996) 719-729.

[S1] P. Sargos, Prolongement méromorphe des séries de Dirichlet associées à des fractions rationnelles de plusieurs variables, Ann. Inst. Fourier 33 (1984), 83-123.

[S2] P. Sargos, Croissance de certaines séries de Dirichlet et applications, J. Reine Angew. Math. 367 (1986), 139-154.

[T] E.C. Titchmarsh, The theory of the Riemann zeta-function, Oxford Sci. Pub., (1986).

[V] I. Vardi, Determinants of Laplacians and multiple gamma functions, SIAM J. Math. Anal. 19(1988), 493-507.

[WY] L. Weng and Y. You, Analytic torsions of spheres, Intern. J. Math. 7 (1996), 109-125. 